\title{
Quantum Hypercomputing and Communications: Overview and Future Applications
}

\author{
LUIGI MAXMILLIAN CALIGIURI \\ Foundation of Physics Research Center (FoPRC) \\ Via Resistenza, 87053 Celico (CS) \\ ITALY \\ ANTONIO MANZALINI \\ TIM \\ Innovation \\ Via Reiss Romoli, 274, 10148 Torino (TO) \\ ITALY
}

\begin{abstract}
Today, like never before, we are witnessing a pervasive diffusion of ultra-broadband fixed-mobile connectivity, the deployment of 5G infrastructures and its integration with Cloud-Edge Computing. Moreover, Artificial Intelligence (AI) is at the center of this Digital Transformation. Sustainability of future scenarios will have to face several techno-economic challenges: the transmission and processing of enormous quantity of data with ultra-low latencies, automation of management processes, strict requirements of resilience, security and privacy, optimization of resources and energy, new markets dynamics. This is requiring a rethinking of the ways of doing computation and communications. As a matter of fact, a second revolution is underway with a growing interest - and several investments from Industries worldwide - and expected potential exploitations for meeting said evolutionary requirements and the sustainability. This paper provides a description of an innovative approach based on evanescent, electromagnetic waves and tunneling photons, for developing quantum computing systems, so called Quantum Hypercomputing. Some examples of potential applications are also provided
\end{abstract}

Key-Words: - Quantum communications; Quantum computing; Evanescent photons; Hypercomputing

Received: April 5, 2021. Revised: September 25, 2021. Accepted: October 10, 2021. Published: October 23, 2021.

\section{Introduction}

A first quantum revolution has already brought to the exploitation of quantum component and devices in our everyday life: micro-electronics components, chipsets, LED and lasers, systems for medical imaging (Nuclear Magnetic Resonance, Positron Emission Tomography), etc. are examples of component, devices and systems whose functioning is based on quantum mechanics principles.

Today, a second revolution is underway. The goal is exploiting the technology advancements in detecting and manipulating quantum objects (e.g., photons, electrons, atoms, molecules). In particular, there are three quantum phenomena, that a second revolution aims at exploiting: superposition, entanglement and measurement. Superposition concerns the property of quantum objects to stay in linear combination of multiple states until they are observed; entanglement is defined as the possibility that two or more quantum objects to stay intrinsically linked, into an intertwined composite state, regardless of how far apart the objects are from one another; measurement regards the collapse and disruption of a quantum state from coherent probabilistic superposition state into a discrete on.

Currently, there is an impressive grow of interests, demonstrated by several investments from public and private organizations, creation of start-ups, activities in standardization bodies (e.g., ETSI, ITU, IETF/IETF, GSMA...). It is expected that within 10 years, the second quantum revolution will generate an impact over many markets, ranging from Telecom and ICT, to Medicine, to Finance, to Transportation, and so on [1].

Four main applications areas have been identified: communications, computing, simulations, sensing and metrology. 


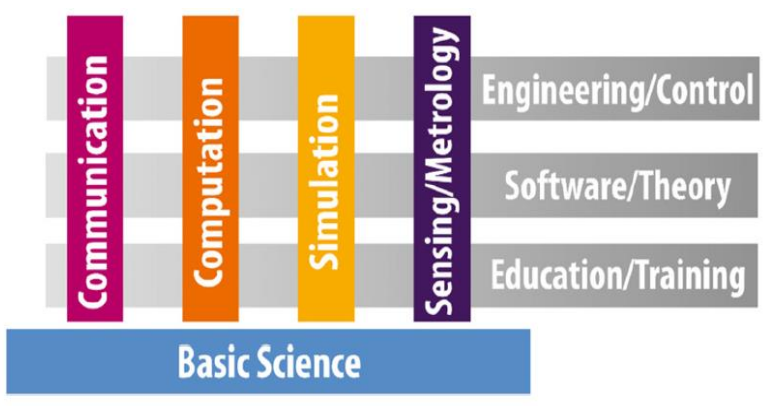

Figure 1. Second quantum revolution: applications areas of quantum technologies (source http:/qurope.eu/h2020/qtflagship/roadmap2016).

The area of quantum communications includes two avenues: 1) the so-called quantum-safe communications and 2) the teleporting of quantum information (e.g., Quantum Internet). The former avenue is about quantum-safe communications which are leveraging on systems such as Quantum Key Distribution (QKD) and Quantum Random Number Generators (QRNG). The latter avenue regards the teleporting of qubits, but this is for longer terms scenarios, such as the Quantum Internet [2], [3].

The avenue of quantum computing aims at speeding up processing of data over classical computers. This may help in solving complex optimization and combinatorial problems (from exponential to polynomial processing time). Today, trapped ions and superconducting qubits seems to be the quantum computing technologies which better satisfy the five required criteria for quantum computing, formerly defined by Di Vincenzo:

- A scalable physical system with well characterized qubits;

- The ability to initialize the state of the qubits to a simple fiducial state;

- Long relevant decoherence times;

- A "universal" set of quantum gates;

- A qubit-specific measurement capability.

The application area of quantum simulations concerns all those applications where wellcontrolled quantum systems are used to simulate the behavior of other systems, which are less accessible and more complex for a direct simulation. Quantum sensing and metrology includes those applications where high sensitivity of quantum systems can be exploited to measure physical properties and timing with more precision (e.g. magnetic and heat sensors, gravimeters, GPS-free navigators, clocks).

The main contributions of this paper include:
- a brief description of the context of the second quantum revolution;

- a description of an innovative approach based on evanescent, electromagnetic (e.m.) waves and tunneling photons, for developing quantum computing system, so called Quantum Hypercomputing;

- an example of some potential applications of Quantum Hypercomputing in a future smart city.

\section{Quantum Computing and Communications}

The second quantum revolution aims at exploiting technology advancements which are leveraging on superposition, entanglement and measurement. Quantum Communications and Computing are the two areas of applications where major impacts are expected for Telecommunications.

Short-term applications will concern quantum security in data exchange, implemented through QKD systems. Furthermore, the development of chipsets for the generation of quantum random numbers QRNG will lead to the development of quantum security applications, including for communication services and the Internet of Things.

Furthermore, the use of quantum computing systems will allow to solve optimization problems with very complex constraints in a much shorter time. Examples of problems that could be solved with quantum computing include: network planning, optimization in the dynamic allocation of network resources and services; optimization of traffic flows; enhancement of the performance of Artificial Intelligence systems; classification of data on large spaces, identification of patterns, or schemes, recurring for applications and services.

The expected benefits are:

- reduction TCO (Total Cost of Ownership) of infrastructures and increased performance: thanks to the use of quantum computing it will be possible to optimize the use of network resources, energy consumption and operating costs;

- increased levels of security in communications and data exchange: thanks to the QKD and QRNG systems it will be possible to provide virtually inviolable cryptographic services as they are based on the paradigms of quantum physics; 
- new services and paradigms of interaction and communication, e.g. Quantum Security as a Service, Quantum Cloud Computing as a Service, etc...

There are some points of attention, such as: the definition of network architectures and the standardization of the interfaces of quantum systems, so that they can be easily integrated, also from the point of view of management and control, in current networks.

\section{A not too visionary "vision": Quantum Hypercomputing}

This section describes the possibility to realize the so-called "hpercomputing", namely the execution of a virtually infinite number of computational steps in a finite time [4].

It is commonly believed that theoretical limitations arising from fundamental physics will broke Moore's law about microprocessor performances in the near future. As shown by Feynman [5], the computational speed is limited by the minimum amount of energy required to transport a bit of information, in an irreversible way, between two devices. This could be a strong limitation even in quantum computation for which computational speed as a function of required energy is limited by energy dissipation during the process.

As already shown by L.M. Caligiuri and coworkers [6-12] a possible way to overcome this limit could be to realize a so-called "accelerated Turing machines", also known as "Zeno machine" (ZM), that is a hypothetical computational device able to perform a countable infinite number of computational steps within a finite time interval and able, for example, to solve the famous "halting problem" (HP) for the TM in a finite interval of time. We can consider a ZM to be identical to a TM with its input, output and storage tapes, executing the first step in $1 / 2$ of a finite time interval $\Delta t$, the second in a $(1 / 4) \Delta t$, the third in a $(1 / 8) \Delta t$ and so on.

An important question is about the existence of a physical process able to perform hypercomputation. In fact, whatever be the notion of "computability" we assume, computation is, in principle, a physically achievable process. In this regard, the concept of a $\mathrm{ZM}$ is thought to contrast with the Heisenberg uncertainty principle since the energy required to perform a calculation exponentially increase as the computational step is accelerated.
For this reason, the ZM has been generally considered, so far, just like a mere mathematical concept unable to be practically realized.

On the other hand, the realization of an hypercomputing quantum system could solve some of the main present-day theoretical and applied issues related to the application of quantum computing schemes to fields like Artificial Intelligence (AI), for example in the realization of quantum neural networks. A proposal in this sense has been recently advanced by L.M. Caligiuri and T. Musha [6-12] according who shown the feasibility of a device like a ZM, by considering the evanescent photons of e.m. field produced inside physical systems made of metamaterials (MTMs) as the result of coherent dynamics of Quantum Vacuum.

\subsection{Evanescent e.m. waves and tunneling photons}

According to classical theory of optics, an evanescent wave is generated at the interface between two media characterized by different refraction index when the incident wave is reflected at an angle $\theta_{1}>\theta_{c}\left(\theta_{c}\right.$ being the so-called "critical angle") $[6,8]$. In this case, despite according to Snell law all the incident energy should be reflected within the first medium, experimentally, an e.m. perturbation, whose propagation is restricted in the neighborhood of the separating surface between two media, is still present in the second medium and is known as "evanescent" wave (EW) whose intensity exponentially decays with the distance from the surface between the two media. A meaningful feature of EW is that it is described by a wave vector $\vec{k}^{T}$ characterized by purely imaginary component.

It is possible to show $[6,13]$ evanescent waves propagating inside a medium con be actually considered, from a quantum viewpoint, as the quantum-tunneling modes of e.m. waves through a potential barrier.

In particular the following relation holds (for simplicity we can consider unidimensional motion):

$$
k^{2}=2 m(E-V)
$$

in which $k$ is the modulus of e.m. wave vector, $m$ the mass of the "corresponding" tunneling particle, $E$ its kinetic energy and $V$ the potential barrier. The value of $k$ is in turn related to the refraction index $n$ of the propagating medium through the formula: 


$$
k^{2}=n^{2} \omega^{2}
$$

where $\omega$ is the angular frequency of the e.m. wave. We see from equation (1) and (2) that, for a particle confined inside a potential wall, namely when $E<V$, the wave numbers in quantum packet are imaginary as well as the values of refraction index. The refraction index in optics plays the same role of the barrier potential in the quantumtunneling phenomenon so that the spreading of e.m. waves across "impenetrable" opaque optical barriers is analogous to the phenomenon of quantum tunneling experienced by particles through a potential barrier. The tunneling of evanescent modes of e.m. waves can be further interpreted, from a quantum viewpoint, as the tunneling of virtual photons $[6,13]$ through the corresponding potential barrier. As already shown [6,13], tunneling photons associated to evanescent field are characterized by a negative square mass of the photons belonging to it namely, in the ordinary metric, by a superluminal group velocity $[6,13,14]$. This meaningful result about the group velocity of evanescent e.m. waves can be easily verified by recalling the Fresnel's theory predicts the $k^{y}$ component of the propagation vector $\vec{k}$ normal to the reflection plane is imaginary while the component $k^{x}$ parallel to the incident plane is larger than $\omega[8]$.

\subsection{Evanescent photons generated in highly coherent states of condensed matter}

Several previous studies $[15,16]$ have shown that in condensed matter, when suitable conditions about matter density and temperature are meet, the coupling between the fluctuations of e.m. quantum vacuum and those of the matter system (considered, for simplicity, as a two-levels system) became so strong to determine a "superradiant" quantum phase transition (SPT) from a so-called "perturbative ground state" (PGS), characterized by the quantum zero point oscillations of e.m. field and matter only, to a "coherent ground state" (CGS) in which both the e.m. and matter field oscillate in phase with each other at a common frequency $\omega_{c o h}$. In the CGS the system experiences a collective common behavior of the quantized e.m and matter fields manifesting itself as a macroscopic quantum object in which its elementary components lose its individuality to become part of a whole e.m. field + matter entangled system, similar, in many regards, to a Bose-Einstein Condensate (BEC). A very remarkably consequence of SPT is the emergence of the so-called "Coherence Domains" (CDs), namely the smallest spatial regions in which the coherent evolution of the e.m. + matter field takes place. Inside CDs the above dynamics determines an extended oscillating polarization field that correlates a high number of elementary matter electric dipoles generating, within every $\mathrm{CD}$, a stable and ordered macroscopic structure.

The photons (also called "superradiant" photons) belonging to the e.m. field coherently interacting with the matter field inside CDs are characterized $[15,16]$ by a frequency $\omega_{c o h}$ of photons lower than the frequency $\omega_{0}$ of free e.m. characterizing the non-coherent state or PGS. The condition $\omega_{c o h}<\omega_{0}$ implies, according to the Einstein - De Broglie relation, the mass belonging to these photons has a purely imaginary value, meaning coherent e.m. field is "trapped" inside CDs, except for an evanescent tail of e.m. field escaping from the CD's boundaries. $[6,15,16]$.

The coherent e.m. field generated inside CDs is also very stable with respect to quantum and thermal fluctuations that characterize the PGS and that make the system to decohere and, for many systems, this stability can even persist at room temperature, as long as the coherent fraction of system components is still sufficiently high. Under such conditions, a number of coherent evanescent photons can be "extracted" from CDs without putting the system out of CGS and "used" to perform quantum hypercomputation $[6,12]$ as will be explained in the following sections.

\subsection{Quantum computation by means of evanescent photons}

As commonly believed, large-scale quantum computers would be able to solve certain problems much more quickly than any classical computer using the best currently known algorithms, like integer factorization using Shor's algorithm or the simulation of quantum many-body systems. As recalled in this section the realization of hypercomputation would boost computational power well beyond the so-called "Turing barrier".

The idea and the ways of exploiting evanescent photons, generated in highly coherent states of condensed matter, and quantum tunnel effect to perform quantum hypercomputation have been proposed by L M. Caligiuri and his co-workers some years ago in a series of recent papers [6-12]. On the other hand, the quantization of evanescent e.m. field [6] precisely allows the evanescent photons to undergo to tunnel effect, like "ordinary" photons. 
The idea of using tunneling photons in signalprocessing devices, although not related to quantum computing, has been already tested in the past [17] allowing for the possibility to realize, for example, all-optical switches on a microscopic scale able to manipulate single electrons, atoms or photons. If the atoms can be localized at distances smaller than the radiation wavelength, they can be coherently coupled by photons realizing an entangling quantum logic gate [6].

This technology could be used to realize, for example, a large-scale computing system containing a large number of small interconnected e.m. ion traps able to perform both memory and logical processing by manipulating atoms by means of the tunneling photons [6]. This kind of computer system will then consist of quantum gates using quantum tunneling photons (QTP) to realize logical operations.

As pointed out by L.M. Caligiuri and T Musha [6], in quantum computer system utilizing evanescent photons, computational gates could be entangled with each other, exerting their influences well outside the extent of the processor. This is due to the evanescent field associated to the coherent e.m. field generated inside every $C D$ in condensed matter whose tail "escapes" from it and is capable to interact with the similar evanescent fields associated to the neighbouring CDs.

As shown by L.M. Caligiuri et al. [6-12] such a technology, using quantum tunneling photons, could be able to achieve much higher computational speed than "usual" quantum computational schemes so far proposed, without incurring in their common and most critical issues as, first of all, the environmental decoherence and the speed limitation due to the energy cost imposed by Heisenberg uncertainty principle as reviewed in the following.

It has been calculated that the minimum time required to perform a single logical operation can be estimated as $[6,8,11]$

$$
\Delta t \approx \frac{\pi N}{2<E>}
$$

where $N$ is the number of computer gates and $\langle E\rangle$ the average energy of the computational system. From equation (3) the time required to perform a single logical operation by using QTP gates can be calculated as $[6,8]$

$$
\Delta t \approx \frac{N}{\beta(\beta-1)} \frac{\pi}{2<E_{*}>}
$$

in which $\left\langle E_{*}\right\rangle$ is the averaged energy for QTP gates, $\beta=v / c, v$ is the speed of the tunneling photon and $c$ the speed of light in vacuum. The uncertainty in the momentum of tunneling photons moving can be given by [6]

$$
\Delta p=\frac{\mu v}{\sqrt{v^{2}-1}}-\hbar \omega
$$

where $\mu$ is the absolute value of the mass for the tunneling photon and $\omega$ its angular frequency. The velocity of the tunneling photon can be then written as $[6,8]$ :

$$
v \approx\left(1+\frac{1}{\sqrt{\omega \Delta t}}\right)
$$

If $d$ is the length of the tunneling barrier, the time required by a photon to tunnel across it across can be roughly estimated as $\Delta t=d / v$, so the velocity of the tunneling photon is given by

$$
v \approx\left(1+\frac{1}{2 \omega d}+\sqrt{\frac{1}{\omega d}+\frac{1}{4 \omega^{2} d^{2}}}\right)
$$

By equating equation (3) to (4) we can calculate the ratio of the minimum energy required for computation by QTP gates and the conventional computation as $[6,8]$

$$
R=\frac{<E_{*}>}{<E>} \approx \frac{1}{\beta(\beta-1)}
$$

in which

$$
\beta \approx 1+\frac{1}{2 \omega d}+\sqrt{\frac{1}{\omega d}+\frac{1}{4 \omega^{2} d^{2}}}
$$

The value of $R$ depends on the tunneling "distance" $d$ that is related to the physical process that generates the evanescent photons. For example, it has been estimated [6] that the use of evanescent photons belonging to infrared region $\left(\lambda \sim 10^{5} \mathrm{~nm}\right)$, corresponding to a tunneling distance $d \approx 10 \mathrm{~nm}$ $[6,8]$, would reduce the energy cost of computation for a computer made of QTP gates of a factor $10^{-6}$ 
with respect to a conventional computer systems. The theoretical analysis just reviewed shows the energy loss of a computational system using tunneling photons is much lower than that of conventional silicon processors.

Moreover, the very high velocity reachable by the tunneling photons, as given by (7), can eventually speed up computational speed significantly because it can:

a) to compensate for interconnection delays inside logic gates, which can never be fully eliminated from any real electronic components;

b) to bring overall transmission rate closer to the ultimate speed limit $[6,8,11]$;

actually resulting in the boost of the speed of a signal traveling on an e.m. wave and in the achievement of high performance computers.

As well-known, one of the most critical issues affecting the practical realization of a large-scale quantum computer is related to the loss of coherence of quantum states that is very fragile and difficult to maintain. In fact, the qubit calculations of the quantum computer are performed while the quantum wave function is in a state of superposition between states that allows it to perform the calculations using both the states $|0\rangle$ and $|1\rangle$ states simultaneously.

The minimum energy required to perform quantum computation for a n-qubits system can be then given by $[6,8]$ :

$$
E_{0} \approx \frac{n \nu_{G}}{T} 2^{n}
$$

where $\nu_{G}$ is the number of gate operations and $T$ the relaxation time of a single qubit. In the same way the minimum energy required to perform quantum computation utilizing evanescent photons particle can be estimated as $[6,8]$ :

$$
E_{0}^{\prime} \approx \frac{n \nu_{G}}{\beta(\beta-1) T} 2^{n}
$$

with $\beta$ given by equation (9). We can calculate the increase in the qubit size $\Delta n$ obtained by using evanescent photons with respect conventional computing by assuming $E_{0}=E_{0}^{\prime}$, and obtaining $[6,8]$ :

$$
\Delta n \approx \frac{\log _{2}[\beta(\beta-1)]}{1+1 / n \log 2}
$$

from which we deduce an important increae of the qubit size of computation when $\beta \geq 1$ as occurs for the evanescent photons. Now we'll see how the use of evanescent photons can overcome the issue of quantum decoherence afflicting conventional quantum computational schemes.

Zurek shown the density matrix $\rho\left(x, x^{\prime}\right)$ of the particle in the position representation evolves by the master equation $[8,18]$

$\dot{\rho}=-i[H, \rho]-\gamma\left(x-x^{\prime}\right)\left(\frac{\partial}{\partial x}-\frac{\partial}{\partial x^{\prime}}\right) \rho-2 m \gamma k_{B} T\left(x-x^{\prime}\right)^{2} \rho$

where $H$ is the particle Hamiltonian, $\gamma$ is the relaxation rate, $k_{B}$ is the Boltzmann constant, $T$ the temperature and $m$ the mass of the field. The offdiagonal peaks of the density matrix, $\rho\left(x, x^{\prime}\right)=\phi(x) \phi^{*}\left(x^{\prime}\right)$ will decay at the rate ( $\left.\Delta x \simeq x^{\prime}-x\right)$

$$
\frac{d}{d t}\left(\rho^{ \pm}\right) \approx \frac{2 \gamma m k_{B} T}{(\Delta x)^{2} \rho^{ \pm}}=\frac{\rho^{+}}{\tau_{D}}
$$

where $\tau_{D}$ is a decoherence time. From (14) we derive the decoherence time in a quantum processor utilizing evanescent photons is obtained as $[6,8]$

$$
\tau_{D}^{\prime} \approx \tau_{R}\left(\frac{1}{\Delta x \sqrt{2 m k_{B} T}}\right)^{2}[\beta(\beta-1)]^{2}
$$

Equation (15) tells us if the error rate is small enough, so that quantum error correction can be used, the decoherence time can be made, by a suitable value of $\beta$, sufficiently longer to allowing the overall quantum calculation to terminate.

As already pointed out above, Hypercomputation, conceptually introduced by Jack Copeland and Diane Proudfoot [4], refers to models of computation that goes beyond, or are incomparable to, Turing computability and includes various hypothetical methods for the computation of nonTuring-computable functions. In this sense a possible hypercomputer would be able to compute functions that a Turing machine cannot and which are, hence, not computable in the Church-Turing sense. 
According to the "classical" model of computation defined by Feynman, the energy required per step of computation is [5]

$$
\text { energy per step }=k_{B} T \frac{f-b}{(f+b) / 2}
$$

where $k_{B}$ is Boltzmann's constant, $T$ is the temperature, $f$ is a forward rate of computation and $b$ is a backward rate. If we suppose there in no energy supply, the parameters $f$ and $b$ remain constant during the calculation and the energy loss per computational step $\Delta E_{i}$ is given by [6-9]:

$$
\begin{gathered}
\Delta E_{1}=E_{0}-E_{1}=(1-k) E_{0} \\
\Delta E_{2}=E_{1}-E_{2}=(1-k) k E_{0} \\
\vdots \\
\Delta E_{n}=E_{n-1}-E_{n}=(1-k) k^{n-1} E_{0}
\end{gathered}
$$

where $k=2(f-b) /(f+b), \quad E_{i}=k^{i} E_{0}$ is the energy for the $\mathrm{i}$-th computational step $(1 \leq i \leq n)$ and $E_{0}=k_{B} T$. According to Lloyd [19], the minimum time required by a quantum system to evolve to an orthogonal state (that we could rouglhy assume as an indication of the smallest computational time for a single logical operation) is given by

$$
\Delta t=\frac{\pi}{2 \Delta E}
$$

where $\Delta E$ is the average energy of the system. Equations (17) and (18) allow to calculate the time required to perform an infinite number of computatiol steps as [6-9]

$$
T=\sum_{n=1}^{\infty} \Delta t_{n}=\frac{\pi}{2 E_{0}} \sum_{n=1}^{\infty} \frac{1}{(1-k) k^{n-1}}
$$

that diverges to infinity if $0<k<1$. This means an accelerated Turing machine cannot be realized by considering ordinary particles due to the constraint imposed by the time - energy uncertainty principle. L.M. Caligiuri and T. Musha shown [6-9] that instead, by considering evanescent photons, the time required to perform an infinite number of computational steps can be written as:

$$
T=\sum_{n=1}^{\infty} \Delta t_{i}=\frac{\pi}{2 E_{0}} \sum_{n=1}^{\infty} \frac{1}{\beta_{n}\left(\beta_{n}-1\right)(1-k) k^{n-1}}
$$

where the value of $\beta$ is now given by

$$
\beta_{n}=\sqrt{1+\frac{\mu^{2}}{E_{n}^{2}}}=\sqrt{1+\frac{\mu^{2}}{k^{2 n} E_{0}^{2}}}
$$

that always converges to a finite value when $0<k<1$.

As for example, in fig. 1 is represented the computational time, calculated by (20), required to perform an infinite number of calculation steps for different values $0<k<1$, in which the horizontal line represents the parameter $\gamma=\mu / E_{0}$ and the vertical one the time to complete the infinite step of calculations, expressed as

$$
T_{n}=\left(\pi / 2 E_{0}\right) \sum_{i=1}^{n} 1 / \beta_{i}\left(\beta_{i}-1\right)(1-k) k^{i-1} .
$$

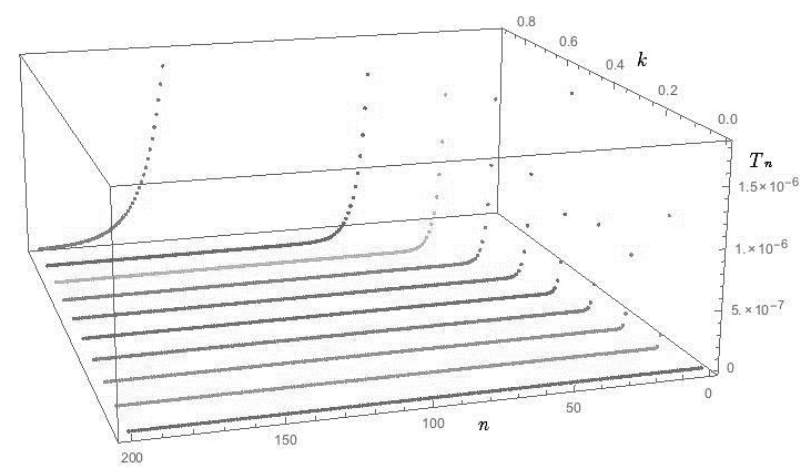

Figure 1. Computational time required required time when using evanescent photons.

These calculations clearly show how an accelerated TM can be in principle realized by using, within the Feynman computational model, evanescent photons instead of "ordinary" ones.

Thus, contrary to the conclusion currently inferred from Feynman model of computation when applied to ordinary particles, the use of evanescent photons particles can overcome the main theoretical questions posed by such model, so allowing the realization of an accelerated TM so opening the doors to the actual design and construction of a quantum hypercomputer based on evanescent photons. 


\section{A novel computer architecture for quantum hypercomputing}

It has been shown by L.M. Caligiuri and T. Musha [6-10] that by exploiting the above dynamics of evanescent tunneling photons, a tubular structure made by metamaterials (MTMs) can achieve quantum bit (qubit) computations on large data sets, allowing high computational performance and very low energy consumption if compared with the conventional silicon processors. On this basis, macroscopic quantum ordered dynamical systems generating evanescent photons inside a computer processor could realize long-range coherence in a computing system.

Metamaterials incorporate structural elements of sub-wavelength sizes, i.e. features that are actually smaller than the wavelength of the waves, giving rise to a negative refractive index. Such materials then allow the creation of "super lenses" that can have a spatial resolution below that of the incident wavelength. It has been shown [20] the use of MTMs can strongly enhance the generation and "propagation" of evanescent photons.

If the inner medium of a MTM-made structure possesses the characteristics of a negative refractive index, the generation of evanescent photons is enhanced, and they propagate without loss according to the properties of the used MTM [20]. A possible idea for realizing a quantum hypercomputer, already presented in some previous research works [6-10], refers to the replacement of electronic components with optical ones, realizing a sort of optical transistors by using materials characterized by a non-linear refractive index. Such an "optical transistor" [21] can be used to implement optical logic gates, which in turn are assembled into the higher-level components of the computer's CPU, such as nonlinear crystals used to manipulate light beams in order to control other ones.

These devices would allow the implementation of the so-called "photonic logic" (PL), namely the use of photons in logic gates such as NOT, AND, OR, NAND, and etc. in which the switching is obtained by using nonlinear optical effects when two or more signals are combined. A possible role of MTMs in realizing PL is to allow an increase of energy from constructive interference, thus enhancing optical nonlinear effects by using semiconductors inside the sub-structure of the MTM itself.

The conceptual scheme of a quantum computer based on evanescent photons dynamics so far emerged is shown in fig. 2 and refers to three main elements [6-10]: 1) an evanescent photon generator, 2) the quantum processor and 3) the holographic memory.

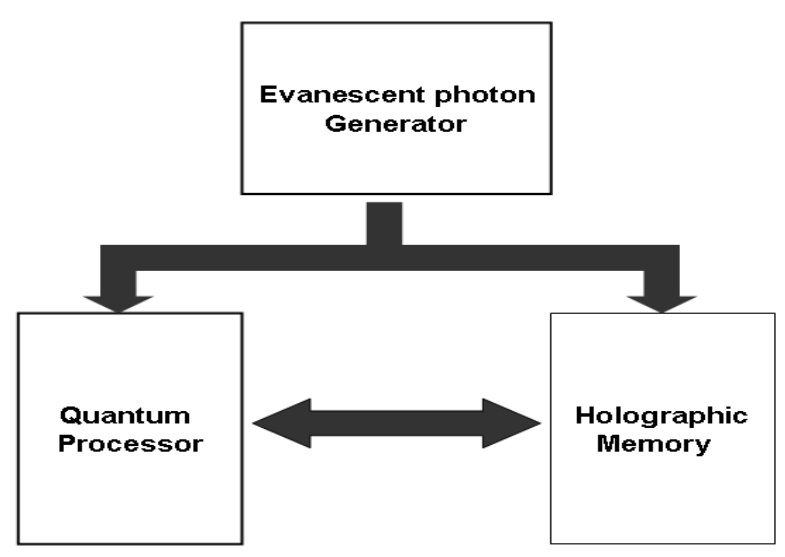

Figure 2. General scheme of a possible quantum computer using evanescent photons.

As previously discussed [6-9], the quantum processor and the holographic memory can be implemented by using the substructures characterizing MTMs inside a tubule-like structural configuration, while the evanescent fields can be generated by considering the quantum dynamics of highly coherent states in suitable condensed matter systems, composed by macroscopic quantum coherent domains (CDs) as discussed in the previous section.

\section{Examples of use-cases and applications}

The ongoing Digital Transformation of telecommunications and Internet will radically increase the flexibility of network and service platforms while ensuring the levels of programmability, reliance and performance required by future $5 \mathrm{G}$ applications (e.g., Internet of Things, 
Tactile Internet, Immersive Communications, Automotive, Industry 4.0, Smart Agriculture, Omics and E-Health, etc).

On the other hand, the management complexity of such future telecommunications infrastructures will overwhelm human-made operations, thus posing urgent needs of designing and deploying management systems with AI capabilities. As a matter of fact, the use of the huge data lake generated by a telecommunications infrastructure will allow automating processes by introducing cognitive capabilities at various levels. Some examples include understanding application needs and automating the dynamic provisioning of services; monitoring and maintaining network state; dynamically allocating virtual network resources and services; ensuring network reliability and enforcing security policies.

Moreover, although we cannot yet fully grasp how pervasive AI will be, it is likely that it will also enable innovative features when provisioning future digital cognitive services for homes, businesses, transportation, manufacturing, and other industry verticals, included the smart cities. At the same time, AI is expected to become increasingly pervasive and, more and more energy-intensive and time-consuming from the processing viewpoint. This might become not easily sustainable when looking at evolution towards 6G. Considering, moreover, that the ongoing Digital Transformation is still laying its foundations on Electronics and the impending end of Moore's Law, it is necessary to pursue a rethinking of the ways of doing computation [22] and communications. The second quantum revolution goes in this direction. It appears reasonable to imagine that, even in the medium term, quantum communications and computing technologies may offer the opportunity of developing new and innovative approaches outperforming the current digital ones: reducing processing and communications latency whilst saving energy at the same time.

Quantum Hypercomputing is a promising approach to meet such requirements.

Let's consider for example the use case of a future smart city. This represents a challenging context as the ever-growing level of complexity of modern cities will require solving, in (almost) real time, combinatorial and local-vs.-global optimization problems with multiple constraints. For instance, one can imagine that every smart car equipped with AI systems capable of solving local transportation problems by constantly scanning a smart radio environment to determine the best route for reaching a destination or whether a car should stop or accelerate at the certain intersection. Such local decisions might not be optimal on a larger scale, so a Quantum Hypercomputing system may optimize the overall city-wide traffic flows in order to send proposals to the cars' drivers to minimize their travel time or to avoid traffic jams and incidents. Quantum Hypercomputing can be used as a sort of brain of the city to solve other combinatorial and optimization problems in a future smart city, e.g., resource and energy savings, emergency, and crises management, etc. We cannot expect to solve complex optimization problems in real time with classical digital computers for questions of processing time and energy consumptions requirements.

As another example, Quantum Twin Computing (QTC) draws inspiration and extend the Digital Twin Computing (DTC) vision, towards 2030. The DTC initiative has been launched by NTT and promoted in [23]. It is well known that a digital twin is a digital representation of features and characteristics of things, such as machinery, appliances, systems, manufacturing processes or even humans. DTC is a paradigm aiming at expanding the above traditional digital twin concept by extending and enhancing communications and interactions in the cyberspace.

In the QTC vision, a Quantum Twin (QT) can be seen as a quantum replica of a living or non-living physical entity, which can be used for various applications purposes: quantum replica means that it is developed using Quantum Hypercomputing technologies. In other words, the Quantum Twin can represent living or non-living physical entity and acting on their behalf in a Quantum Hypercomputing space. A QT can obviously embed AI capabilities and can interact with other QTs.

Similarly, QTC leverages four layers, enabled by a progressive exploitation of Quantum Hypercomputing technologies making it possible to transport, store and elaborate digital and even quantum information.

Quantum interaction layer collecting the physical-world data required to generate a quantum twin, and providing feedback to the physical world from the applications;

- Quantum twin layer generating and maintaining quantum twins by using data received from the quantum interaction layer;

- Quantum world presentation layer providing the framework to invoke 
quantum twin operations using quantum twins stored in the Quantum twin layer; Application layer for developing QTC applications using the quantum world presentation layer.

\section{Conclusions}

Today, like never before, we are witnessing a pervasive diffusion of ultra-broadband fixed-mobile connectivity, the deployment of Cloud-native 5G network and service platforms and a wide adoption of Artificial Intelligence.

The technologies like Software Defined Network (SDN) and Network Function Virtualization (NFV) are offering the opportunity of designing and operating $5 \mathrm{G}$ infrastructures with unprecedent flexibility. In fact, an orchestrated use of Cloud, Edge-Fog computing and network virtual resources can deliver a continuum of capabilities, functions and micro-services...

Sustainability of future scenarios will have to face several techno-economic challenges: the transmission and processing of enormous quantity of data with ultra-low latencies, automation of management and control processes, the fulfilment of the strict requirements of resilience, security and privacy, optimization of energy consumption, new markets dynamics. This is requiring a rethinking of the ways of doing computation and communications. As a matter of fact, a second revolution is underway with a growing interest - and several investments from Industries worldwide and expected potential exploitations for meeting said evolutionary requirements and the sustainability.

This paper provides offers the description of an innovative approach based on evanescent, e.m. waves and tunneling photons, for developing quantum computing systems, so called Quantum Hypercomputing. Such an innovative model of quantum computation could represent the starting point for the design of a novel computer system which would be, in principle, very more powerful than the quantum computers so far proposed and realized, as well as unaffected by the main critical issues limiting them as, for example, environmental decoherence and energy consumption and whose impact on technology would be nowadays unimaginable. An example of some potential applications of Quantum Hypercomputing in a future smart city is eventually provided

\section{References:}

[1] Manzalini, A. Quantum communications in future networks and services. Quantum Reports 2020, 2(1), 221-232.

[2] Cacciapuoti, A. S.; Caleffi, M.; Van Meter, R.; \& Hanzo, L. When entanglement meets classical communications: Quantum teleportation for the quantum Internet. IEEE Transactions on Communications, 2020.

[3] Cacciapuoti, A. S.; Caleffi, M.; Tafuri, F.; Cataliotti, F. S.; Gherardini, S.; \& Bianchi, G. Quantum internet: Networking challenges in distributed quantum computing. IEEE Network 2019, 34(1), 137-143.

[4] Copeland, B. J.; Proudfoot ,D. Alan Turing's Forgotten Ideas in Computer Science. Scientific American. 1999, 99, 103.

[5] Feynman, R. P. Simulating physics with computers. Int. J. of Theor. Phys. 1982, 21, 467-488.

[6] Caligiuri, L.M.; Musha T. The Superluminal Universe: from Quantum Vacuum to Brain Mechanism and Beyond; NOVA Science Publishers Inc.: New York, USA, 2016.

[7] Caligiuri, L. M.; Musha, T. Superluminal Photons Tunneling through Brain Microtubules Modeled as Metamaterials and Quantum Computing. In Advanced Engineering Materials and Modeling; Tiwari, A., Arul Murugan, N., Ahula, R., Eds.; Wiley Scrivener Publishing LLC: Beverly MA, USA, 2016; pp. 291-333.

[8] Caligiuri, L.M.; Musha, T. Quantum hypercomputing by means of evanescent photons, J. Phys.: Conf. Ser. 2019, 1251, 012010.

[9] Caligiuri, L.M.; Musha, T. Accelerated Quantum Computation by means of Evanescent Photons and its Prospects for Optical Quantum Hypercomputers and Artificial Intelligence. In Proceedings of the 2019 International Conference on Engineering, Science, and Industrial Applications (ICESI), Tokyo, Japan, 22-24 August 2019.

[10] Caligiuri, L.M. Quantum (Hyper)Computation by means of Water Coherent Domains - Part I: The Physical Level. In Frontiers in Quantum Computing; Caligiuri, L.M., Ed.; NOVA Science Publishers Inc.: New York, USA, 2020; pp.1-37.

[11] Caligiuri, L.M. Quantum (Hyper)Computation by means of Water Coherent Domains - Part II: The Computational Level. In Frontiers in Quantum Computing; Caligiuri, L.M., Ed.; NOVA Science Publishers Inc.: New York, USA, 2020; pp.57-102. 
[12] Caligiuri, L.M. Accelerated quantum computation based on quantum coherent dynamics of evanescent photons in liquid water. In Intelligent Computing; Arai, K., Ed.; Springer Nature Switzerland AG: Cham, Switzerland, 2022; pp. 169-187.

[13] Stahlhofen, A. A.; Nimitz, G. Evanescent modes are virtual photons. Europhys. Lett. 2006, 76, 189-195.

[14] Caligiuri, L.M.; Musha, T. A new quantumrelativistic model of tachyons, J. Phys.: Conf. Ser. 2019, 1251, 012009.

[15] Preparata, G. QED Coherence in Matter; World Scientific Publishing Co. Pte. Ltd.: Singapore, London, New York, 1995; pp. 25-63.

[16] Caligiuri, L.M. The Origin of Inertia and Matter as a Superradiant Phase Transition of Quantum Vacuum. In Unified Field Mechanics; Amoroso, R.L., Kauffman, L. H., Rowlands, P., Eds.; World Scientific Publishing Co. Pte. Ltd.: Singapore, London, New York, 2016; pp. 374-395.

[17] Smolyyaninov, I. I.; Zayats, A. V.; Gungor, A.; Davis, C. C. Single Photon Tunneling. http://arxiv.org/PS-cache/condmat/pdf/0110/0110252.pdf.

[18] Zurek, W. H. Decoherence and the Transition from Quantum to Classical - Revisited. Los Alamos Science. 2002, 27, 2-25.

[19] Llyod, S. Ultimate physical limits to computation. Nature. 2000, 406, 1047-1054.

[20] Baena, J. D.; Jelinek, L.; Marwues, R.; Medina, F. Near-perfect tunneling and amplification of evanescent electromagnetic waves in a waveguide filled by a metamaterial: Theory and experiments. Phys. Rev. E. 2005, 72, 075116.

[21] Jain, K.; Pratt, G. W. Jr. Optical transistor. Appl. Phys. Lett. 1976, 28, 719-720.

[22] Manzalini, A. Complex Deep Learning with Quantum Optics. Quantum Rep. 2019, 1, 107118.

[23] Innovative Global Wireless Optical Network Global Forum. Available online: https://iowngf.org/ (accessed on 31st July 2021).

\section{Contribution of individual authors to the creation of a scientific article (ghostwriting policy)}

Author Contributions: Please, indicate the role and the contribution of each author:

Example

Luigi Maxmilian Caligiuri carried out the quantum hypercomputing theory and the conclusions.

Antonio Manzalini has implemented the general part of the paper and the conclusions.

\section{Creative Commons Attribution}

\section{License 4.0 (Attribution 4.0 \\ International , CC BY 4.0)}

This article is published under the terms of the Creative Commons Attribution License 4.0 https://creativecommons.org/licenses/by/4.0/deed.en US 